\title{
Impurity ion flow and temperature measured in a detached divertor with externally applied non-axisymmetric fields on DIII-D
}

\author{
A.R. Briesemeister ${ }^{a^{*}}$, R.C. Isler ${ }^{a}$, S.L. Allen ${ }^{b}$, J.-W. Ahn ${ }^{a}$, A.G McLean ${ }^{b}$, \\ E.A. Unterberg ${ }^{\mathrm{a}}$, D.L. Hillis ${ }^{\mathrm{a}}$, M.E. Fenstermacher ${ }^{\mathrm{b}}$, and W.H. Meyer ${ }^{\mathrm{b}}$ \\ ${ }^{a}$ Oak Ridge National Laboratory, P.O. Box 2008, Oak Ridge, Tennessee 37831, USA \\ ${ }^{b}$ Lawrence Livermore National Laboratory, 700 East Ave, Livermore, California 94550, USA
}

\begin{abstract}
Externally applied non-axisymmetric magnetic fields are shown to have little effect on the impurity ion flow velocity and temperature as measured by the multichord divertor spectrometer in the DIII-D divertor for both attached and detached conditions. These experiments were performed in $\mathrm{H}$-mode plasmas with the grad-B drift toward the target plates, with and without $n=3$ resonant magnetic perturbations (RMPs). The flow velocity in the divertor is shown to change by as much as $30 \%$ when deuterium gas puffing is used to create detachment of the divertor plasma. No measurable changes in the C III flow were observed in response to the RMP fields for the conditions used in this work. Images of the C III emission are used along with divertor Thomson scattering to show that the local electron and C III temperatures are equilibrated for the conditions shown.
\end{abstract}

Abstract length (150 words): 141 currently 
PACS: 52.30.-q, 52.55.Fa, 52.70.Kz, 52.55.Rk

JNM keywords: C0100 Carbon, A0700 Analytical Instruments and Methods

PSI-21 keywords: Plasma flow, Spectroscopy, DIII-D, Detachment, Divertor plasma,

Divertor diagnostic, Carbon-based materials

*Corresponding and presenting author address: c/o General Atomics, P.O. Box 85608, San Diego, California 92186-5608, USA

*Corresponding and presenting author e-mail: breisemeister@fusion.gat.com 


\section{Introduction}

Understanding and modeling divertor plasmas is pivotal for optimizing the design of future fusion reactors where large heat loads will need to be reliably distributed. Impurities play an important role in determining how much power is radiatively dissipated from the plasma and where this radiation occurs. In DIII-D it has been shown that carbon radiates as much and in some cases more power than deuterium [1]. Correctly modeling carbon radiation will therefore be important for predicting plasma conditions in the DIII-D divertor especially for detached conditions, where a significant fraction of the power which enters the scrape off layer (SOL) is radiated before reaching the divertor target plates.

Resonant magnetic perturbations (RMPs) may be used to suppress or mitigate edge localized modes (ELMs) in ITER where it will be necessary to achieve detachment to control heat loads on the divertor plates. It is therefore important to understand what effects RMPs have on detachment. It has been shown in NSTX [2] that RMPs increase the amount of gas needed to achieve divertor detachment. Similar results were seen in DIII-D [3], where applying RMPs caused a reduction in the pedestal density and promoted attachment of the inner divertor leg. Subsequent gas puffing to increase the pedestal density caused "early" detachment of the inner leg at a pedestal density below the value needed to achieve detachment without RMPs. The mechanisms causing these changes are not well understood.

Modifications to the ion flow structure are among the changes that are predicted to occur in response to RMPs. On the open field lines within the divertor large flow velocities can develop as a result of the Bohm sheath criterion at the plasma/wall 
interface [4]. RMPs may lead to the formation of magnetic lobe structures which alter the connection length to the divertor targets [5]. In response to these magnetic perturbations, closely spaced regions of counter streaming flows are predicted to form causing changes in the ion transport within the divertor and potentially changing detachment onset [6].

\section{Experimental setup}

In this work two plasma discharges, shown in Fig. 1, are examined. A discharge in which an axisymmetric magnetic configuration was used is compared to a discharge where the I-coils, shown in Fig. 2, were used to create both even and odd n=3 RMPs. Deuterium puffing, shown in Fig. 1(a), was used to cycle the outer strike point into and out of detachment. Evidence of the detachment of the outer strike point can be seen in the electron temperature measured by the divertor Thompson scattering (DTS) system [7], shown in Fig. 1(e), which drops below $2 \mathrm{eV}$ shortly after the onset of strong gas puffing. This drop in electron temperature is accompanied by a rise in the electron density, shown in Fig. 1(d), measured at the same location.

Applying RMPs did not cause significant changes in the pedestal and divertor plasmas, likely as a result of the relatively high densities used. In the case where RMPs were used, the detachment onset appears to be slightly delayed relative to that in the axisymmetric case. However, the most pronounced difference in the detachment for the two cases is seen before the coils were energized, around 3.2 seconds into the shot. Comparisons of the two cases is further complicated by frequent ELMs, which cause significant perturbations to the DTS measurements. Neither ELM suppression nor mitigation was seen when the RMPs were applied for these cases. ELM suppression in DIII-D is generally only seen when the density at the top of the pedestal $\left(\mathrm{n}_{\mathrm{e} \text { ped }}\right)$ is below 
about $2.5 \times 10^{19} \mathrm{~m}^{-3}$, [3] which is much lower than the pedestal density, shown in Fig. 1(c), used in this experiment to achieve detachment of both strike points.

The primary operating parameters were held fixed for both cases with injected neutral beam power $\mathrm{P}_{\text {ing }}=5.5 \mathrm{MW}$, upper triangularity of 0.21 , lower triangularity of 0.48 , plasma current $\mathrm{I}_{\mathrm{p}}=1.3 \mathrm{MA}$ and toroidal field $\mathrm{B}_{\mathrm{t}}=2.0 \mathrm{~T}$ with $\mathrm{q}_{95}=3.78$. As shown in Fig. 2, for the magnetic configuration used, the outer strike point is on the divertor shelf near the lowest divertor Thomson scattering measurement point.

\section{Spectroscopic instrumentation and views}

Measurements of the C III flows are made using the MDS [8], a 1.3 m Czerny-Turner spectrometer equipped with a 1200 grooves $/ \mathrm{mm}$ grating with an $0.12 \AA$ bandpass and

resolving power of $6.4 \times 10^{4}$. The $4860 \AA \mathrm{C}$ III multiplet and a $200 \mathrm{~ms}$ integration time were used for the measurements shown in this work.

The poloidal projections of the three tangential views used to measure the flow velocity parallel to the magnetic field are shown in Fig. 3, labelled T2, T3 and T4. Because the ion flow velocities in some regions of the diverter approach the ion thermal velocity, changes in the velocity along the line of sight can contribute to the width of the measured emission lines, making it difficult to determine the ion temperature using the tangential views [9]. The ion temperature is measured using the two vertical views labelled L4 and L5 in Fig. 3, which are approximately normal to the magnetic field lines and therefore do not see significant broadening from flow changes.

Because the shape of the multiplet measured by the spectrometer depends on the Zeeman splitting of its components, the magnetic field strength at the location of the emitted light can be determined and used to constrain the spatial origin of the flow 
measurements. The spatial localization of C III within the divertor can be seen in the inversions of image captured using a camera viewing the divertor through an optical filter with a transmission band centred at $4650 \AA$ [10] shown in Fig. 4. As shown in Fig. 4(a), for the outer divertor leg C III emission is concentrated near the strike point indicating that the outer leg is attached. C III for the inner leg is closer to the x-point, indicating that the inner leg is detached. The image in Fig. 4(b) was taken during a high gas puffing phase and shows that the C III emission is primarily localized above the x-point, indicating that both legs are detached.

Comparing Fig. 3 and Fig. 4 it can be seen that the tangential views pass through inboard and outboard regions where C III is present. To account for this, two fit components with independent velocity, temperature, Zeeman broadening and relative strength are used in fitting each spectrum. As an example a measured spectrum and two component fit is shown in Fig. 5. The fitting technique used is described in more detail by Isler [11].

\section{C III flow measurements}

In response to higher deuterium puffing and enhanced detachment, the C III flow is measured to change by as much as $5 \mathrm{~km} / \mathrm{s}$. Fig. 6 shows that the inboard components of the flow move significantly faster than the outboard components through the entire shot. Most of the measurements show a slight increase in the flow velocity during detachment, but the inboard components measured by T3 and T4 show a reduction in flow Detailed measurements and analysis of changes in the local densities and temperatures would be needed to understand these velocity changes during detachment. 
The inboard and outboard components of the flow move in opposite directions as a result of the magnetic geometry within the divertor. Because of the Bohm sheath criteria, the ions tend to move in the direction of shorter distance along the open field lines to the target. The poloidal magnetic field points towards the target on the outboard side and away from the target on the inboard side. As a result the flows tend to travel along the magnetic field on the outboard side and in the opposite direction of the magnetic field on the inboard side. The sign convention used in Fig. 6 was defined relative to the observer, with a positive velocity indicating a counter-clockwise flow. For the magnetic geometry used in the experiment the positive flow seen for the inboard component and the negative flow seen for the outboard component indicate that both components were moving towards their respective targets.

Little change was seen when the flow measured with RMPs are compared to that in the axisymmetric case. The inboard flow components for each of the views measured in the axisymmetric case and the case where RMPs were used for portions of the shot are shown in Fig. 7. The velocities measured by views T4 and T3 are nearly identical for the two cases. When compared to the purely axisymmetric case, the velocity measured by $\mathrm{T} 2$ is slightly higher and shows a smaller response to the transition to attachment in the case where RMPs were used. Difference exist in the measurements from view T2 before 3.5 seconds, when the coils were energized, indicating they may not be caused by the RMPs.

\section{Strong thermal coupling of the electrons and ions}


As shown in Fig. 8 the measured temperature of the C III ions remains fixed at about $15 \mathrm{eV}$ not only with and without RMPs but also shows no change from attached to detached conditions. Examining Fig. 9, which shows the electron temperature at different distances above the target measured by Thomson scattering, it can be seen that when the plasma is attached the electron temperature just above the target is approximately $15 \mathrm{eV}$. During detachment the electron temperature drops and the location at which the electron temperature is about $15 \mathrm{eV}$ moves up to $\mathrm{Z}=-1.14 \mathrm{~m}$. Comparing this with Fig. 3 it can be seen that the region with the highest $\mathrm{C}$ III density tracks the region where $\mathrm{T}_{\mathrm{e}} \sim 15 \mathrm{eV}$. The fact that the C III temperature remains fixed and approximately equal to the local electron temperature indicates that the electrons and carbon ions are strongly coupled for these plasmas.

\section{Conclusion}

Strong C III flows moving towards the divertor plates were measured during both attached and detached conditions. The temperature of the C III ions was shown to remain fixed at about $15 \mathrm{eV}$, strongly coupled to the electron temperature under all conditions examined. It was shown that in the high density conditions used in this paper it is possible to add non-symmetric magnetic fields without significantly perturbing impurity ion flows in the divertor.

\section{Acknowledgment}

This work was supported in part by the U.S. Department of Energy under DE-AC0500OR22725, DE-AC52-07NA27344, and DE-FC02-04ER54698. DIII-D data shown in 
this paper can be obtained in digital format by following the links at https://fusion.gat.com/global/D3D DMP. 


\section{References}

[1] R.C. Isler, et al., Phys. Plasmas 4 (1997) 355.

[2] J.-W. Ahn, et al., Plasma Phys. Control. Fusion 56 (2014) 015005.

[3] T.W. Petrie, et al., Nucl. Fusion 51 (2011) 073003.

[4] P.C. Stangeby, The Plasma Boundary of Magnetic Fusion Devices, Institute of Physics Pub., Bristol; Philadelphia, 2000.

[5] A. Wingen, et al., Phys. Plasmas 16 (2009) 042504.

[6] Y. Feng, et al., Plasma Phys. Control. Fusion 53 (2011) 024009.

[7] T.N. Carlstrom, et al., Rev. Sci. Instrum. 68 (1997) 1195.

[8] N.H. Brooks, et al., Rev. Sci. Instrum. 63 (1992) 5167.

[9] R.C. Isler, et al., Phys. Plasmas 6 (1999) 541.

[10] M.E. Fenstermacher, et al., Rev. Sci. Instrum. 68 (1997) 974.

[11] R.C. Isler, et al., J. Nucl. Mater. 266-269 (1999) 376. 


\section{Figure captions}

Fig. 1. (a) The deuterium gas puff used to detach the divertor is shown along with the coil current for one of the (b) lower I-coils used for the shot with RMPs (red dashed lines), but not for the axisymmetric case (blue solid lines). (c) The electron density at the top of the pedestal is shown along with (d) the electron temperature and (c) electron density measured by DTS at $\mathrm{R}=1.49 \mathrm{~m}, \mathrm{Z}=-1.23 \mathrm{~m}$.

Fig. 2. The magnetic surfaces are shown along with the locations of the upper and lower I-coils used to create the RMPs.

Fig. 3. The poloidal projections of the tangential and vertical views used in this work are shown along with the separatrix and outline of the graphite walls. The x's show the location of the divertor Thomson scattering measurements.

Fig. 4. Images of the CIII emission obtained from inverting the tangential TV images of an (a) attached and (b) detached phase of the shot.

Fig. 5. The dots show a typical measured spectrum while the solid line shows the total fit to that spectrum. The dashed lines show the two independent components of the fit.

Fig. 6. The C III flow velocities measured by view T2 (solid lines), T3 (dashed line) and T4 (dotted line) are shown for times when the plasma is both attached and detached (highlighted). The flow components measured in the inboard portion of the plasma have positive velocity while the outboard components are negative. 
Fig. 7. The inboard component of the C III flow velocities measured by the three views is shown. The solid lines show the case where no 3D fields were used and the dashed line shows the case where 3D fields were applied for some portions of the shot. The times ranges at which different coil parities were used are shown at the bottom of the plot.

Fig. 8. The temperature of the C III ions measured by the two lower viewing chords shown in Fig. 1 are shown for the case where 3D fields were applied (dashed lines) and the case with no 3D fields (solid lines).

Fig. 9. The electron temperature profile measured at different locations above the target plate is show for both attached (circles) and detached (x's) conditions. 


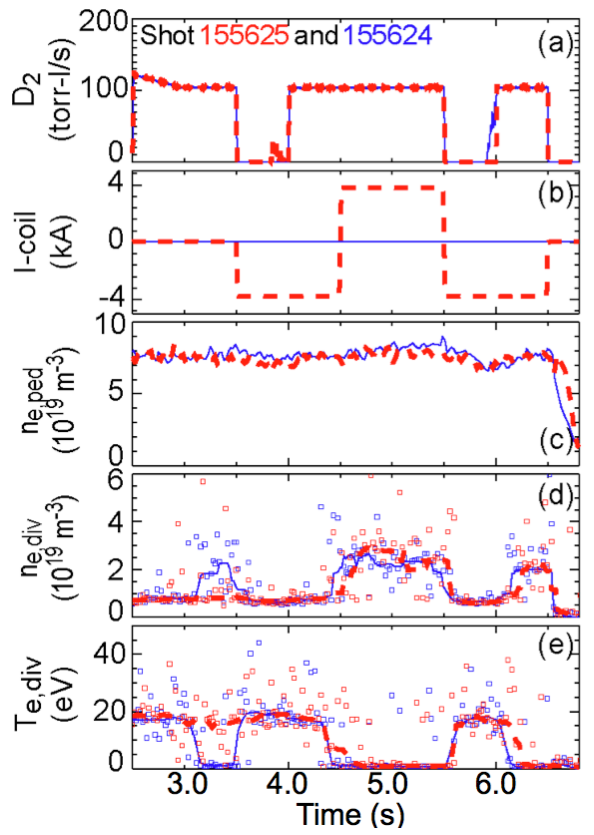

Fig. $1 \quad(85 \mathrm{~mm})$ 


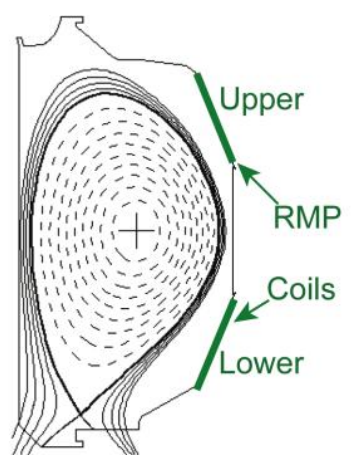

Fig. $2 \quad$ (45 mm) 


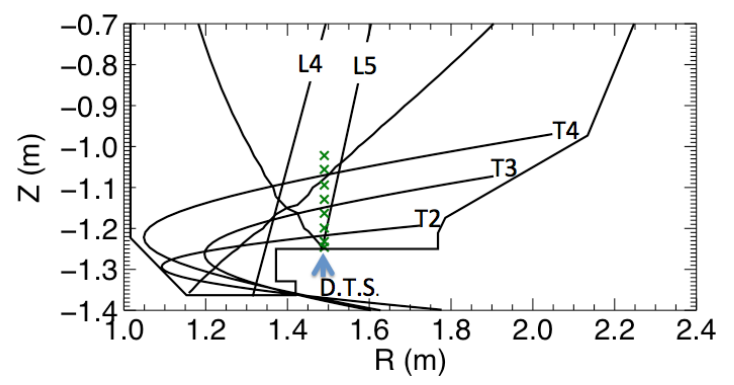

Fig. $3 \quad$ (42 mm) 

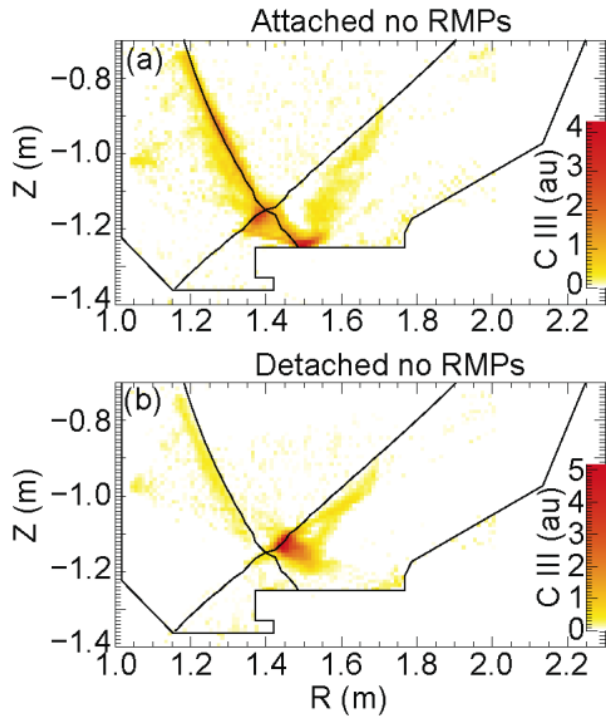

Fig. $4 \quad(72 \mathrm{~mm})$ 


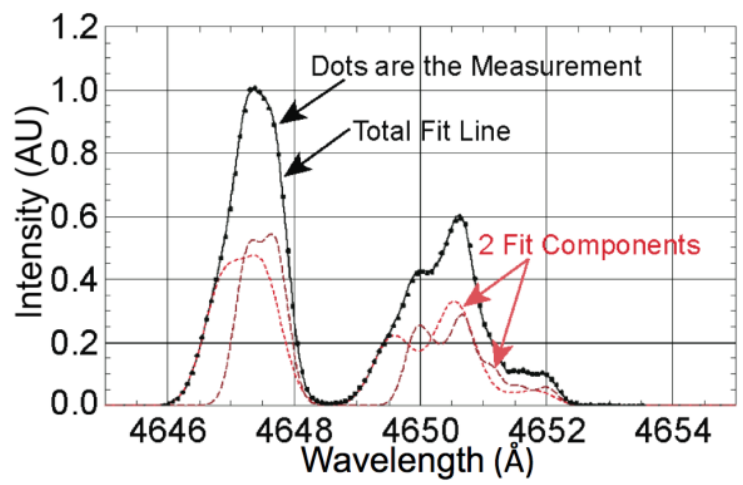

Fig. $5 \quad$ (49 $\mathrm{mm})$ 


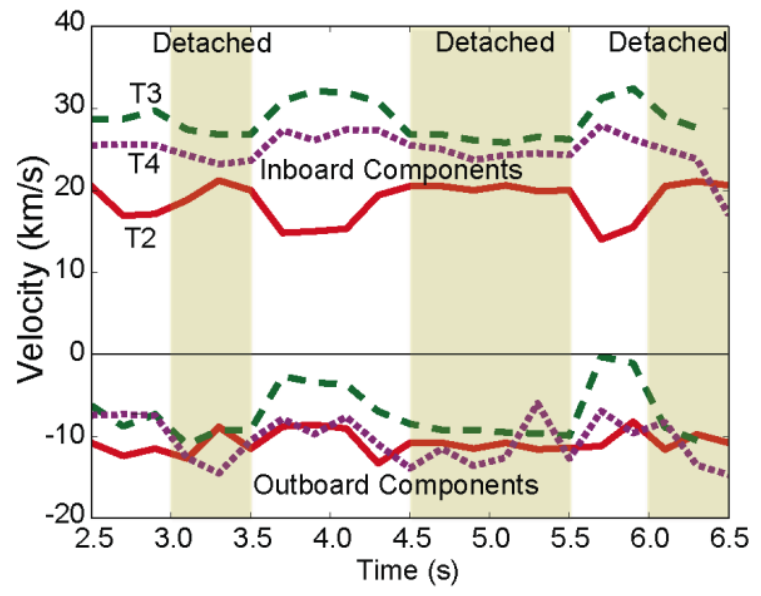

Fig. $6 \quad$ (59 mm) 


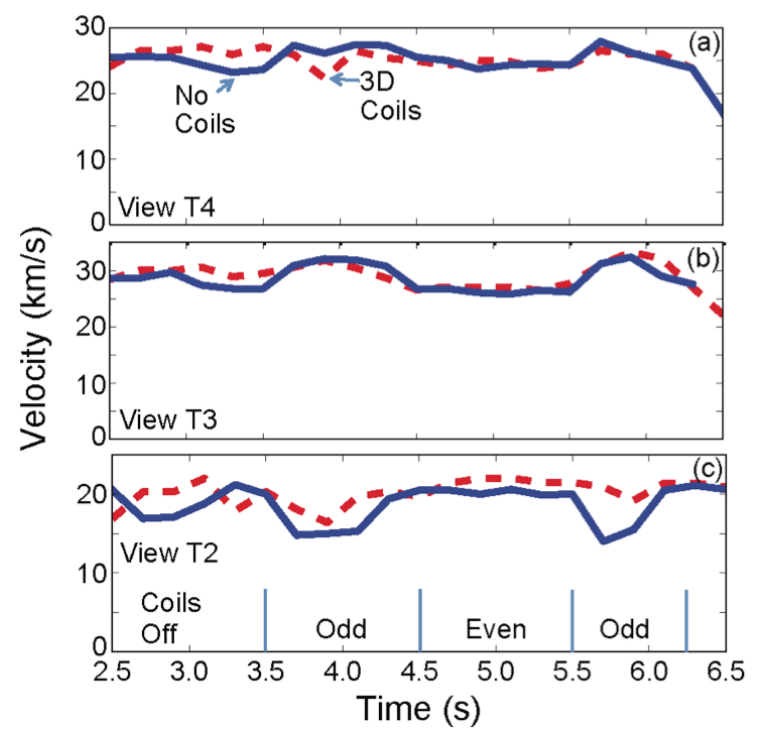

Fig. $7 \quad(74 \mathrm{~mm})$ 

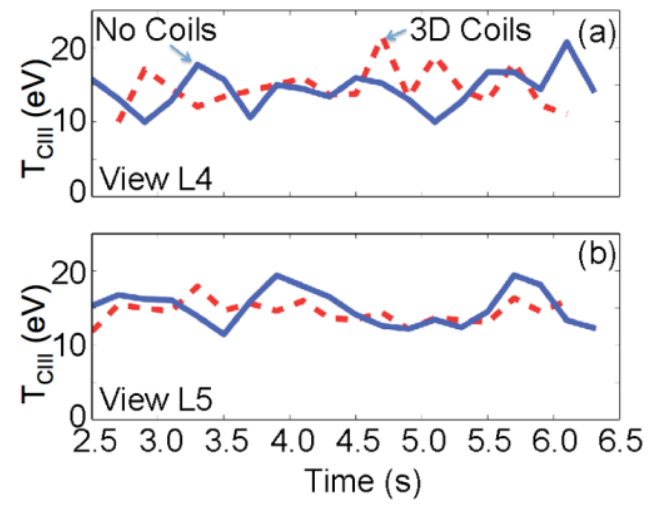

Fig. $8 \quad(51 \mathrm{~mm})$ 


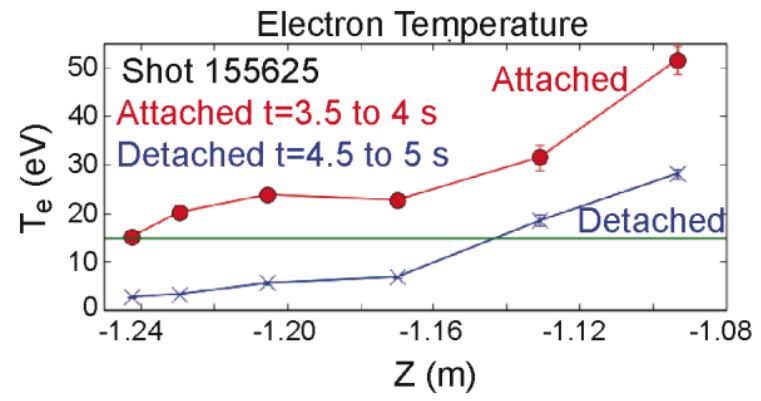

Fig. $9 \quad$ (40 mm) 